TIFR/TH/00-28

hep-lat/0006015

\title{
Suppressing monopoles and vortices : A possibly smoother approach to scaling?
}

\author{
Rajiv V. Gavai円 \\ Department of Theoretical Physics \\ Tata Institute of Fundamental Research \\ Homi Bhabha Road \\ Mumbai 400005, India
}

\begin{abstract}
Suppressing monopoles and vortices by introducing large chemical potentials for them in the Wilson action for the $S U(2)$ lattice gauge theory, we study the nature of the deconfinement phase transition on $N_{\sigma}^{3} \times N_{\tau}$ lattices for $N_{\tau}=4,5,6$ and 8 and $N_{\sigma}=8-16$. Using finite size scaling theory, we obtain $\omega \equiv \gamma / \nu=1.93 \pm 0.03$ for $N_{\tau}=4$, in excellent agreement with universality. Corresponding determinations for the $N_{\tau}=5$ and 6 lattices are also found to be in very good agreement with this estimate. The critical couplings for $N_{\tau}=$ 4, 5, 6 and 8 lattices exhibit large shifts towards the strong coupling region when compared with the usual Wilson action, and suggest a lot smoother approach to scaling.
\end{abstract}

\footnotetext{
${ }^{1}$ gavai@tifr.res.in
} 


\section{INTRODUCTION}

Quantum field theories need regularization schemes to control divergences. The regularization schemes, many different types of which have been used in performing calculations, do not themselves affect physics in any manner, as they are eliminated at the end of all calculations. Long distance physics, such as confinement of quarks in quantum chromodynamics or determination of the hadronic spectrum, is conveniently studied using the lattice regularization. There is a lot of freedom in defining a lattice field theory. In particular, a variety of different choices of the lattice action correspond to the same quantum field theory in the continuum. While many numerical simulations have been performed for the Wilson action [1] for the gauge theories, other choices, some motivated by the desire to find a smoother continuum limit, have also been used. These actions differ merely by irrelevant terms in the parlance of the renormalization group: in the naive classical continuum limit of $a \rightarrow 0$, they all reduce to the same Yang-Mills action and the differing terms are of higher order in $a$.

Investigations of the deconfinement phase transition for mixed actions, obtained by extending the Wilson action by addition of an adjoint coupling

term, showed [2, 3, 田, 5] surprising challenges to the above notion of universality. These actions 6, 7] are

$$
S_{B C}=\sum_{P}\left(\beta\left(1-\frac{1}{2} \operatorname{Tr}_{F} U_{P}\right)+\beta_{A}\left(1-\frac{1}{3} \operatorname{Tr}_{A} U_{P}\right)\right),
$$

and

$$
S_{V}=\sum_{P}\left(\frac{1}{2}\left(\beta+\beta_{V} \sigma_{P}\right) \operatorname{Tr}_{F} U_{P}\right)
$$

Here $U_{P}$ denotes the directed product of the basic link variables which describe the gauge fields, $U_{\mu}(x)$, around an elementary plaquette $P$. $F$ and $A$ denote that the traces are evaluated in the fundamental and adjoint representations respectively and the formula $\operatorname{Tr}_{A} U=\left|\operatorname{Tr}_{F} U\right|^{2}-1$ is used. $\sigma_{P}$ are $Z_{2}$ plaquette fields associated with the plaquette $\mathrm{P}$ and the partition function in the Villain case of eq.(2) has a sum over all possible values for each of them. 
The first term in both the equations describes the standard SU(2) Wilson action whereas the second term adds an adjoint $\mathrm{SO}(3)$ action. For zero adjoint coupling, i.e, for the Wilson action, several finite temperature investigations have shown the presence of a second order deconfinement phase transition. Its critical exponents have been shown [8] to be in very good agreement with those of the three dimensional Ising model, as conjectured by Svetitsky and Yaffe 9]. The verification of the universality conjecture strengthened our analytical understanding of the deconfinement phase transition which, however, came under a shadow of doubt by the results for the mixed actions. Following the deconfinement phase transition into the extended coupling plane by simulating these actions at finite temperature, it was found on a range of temporal lattice sizes for both actions (11) and (2) that:

a] The deconfinement transition was of second order, and in agreement with the conjectured universality exponents, for small values of the adjoint coupling. It became definitely of first order for large values $\beta_{A}$ or $\beta_{V}$.

b] The deconfinement order parameter acquired a nonzero value discontinuously at the transition point for large adjoint couplings.

c] There was no evidence of any other separate bulk transition at those large adjoint couplings, as expected from the results of Refs. [6, 7]. In fact, simulations on larger symmetric lattices even suggested[10] a lack of a bulk phase transition at that adjoint coupling where a first order deconfinement transition for a lattice of temporal size four was clearly seen.

Recently it was shown[11, 12] that suppression of some lattice artifacts such as $Z_{2}$ monopoles and vortices do restore the universality for the action (2): no first order deconfinement transition was found in the entire coupling plane in that case. In this paper, we address this question for the action (1) in the same manner and find that unlike the Villain action, one gains an additional bonus. The approach to scaling seems to become smoother than that for the original Wilson action. The organization of the paper is as follows: In section 2 we define the action we investigate and briefly recapitulate the definitions of various observables used and their scaling laws. 
We present the detailed results of our simulations in the next section and the last section contains a brief summary of our results and their discussion.

\section{THE MODEL AND THE OBSERVABLES}

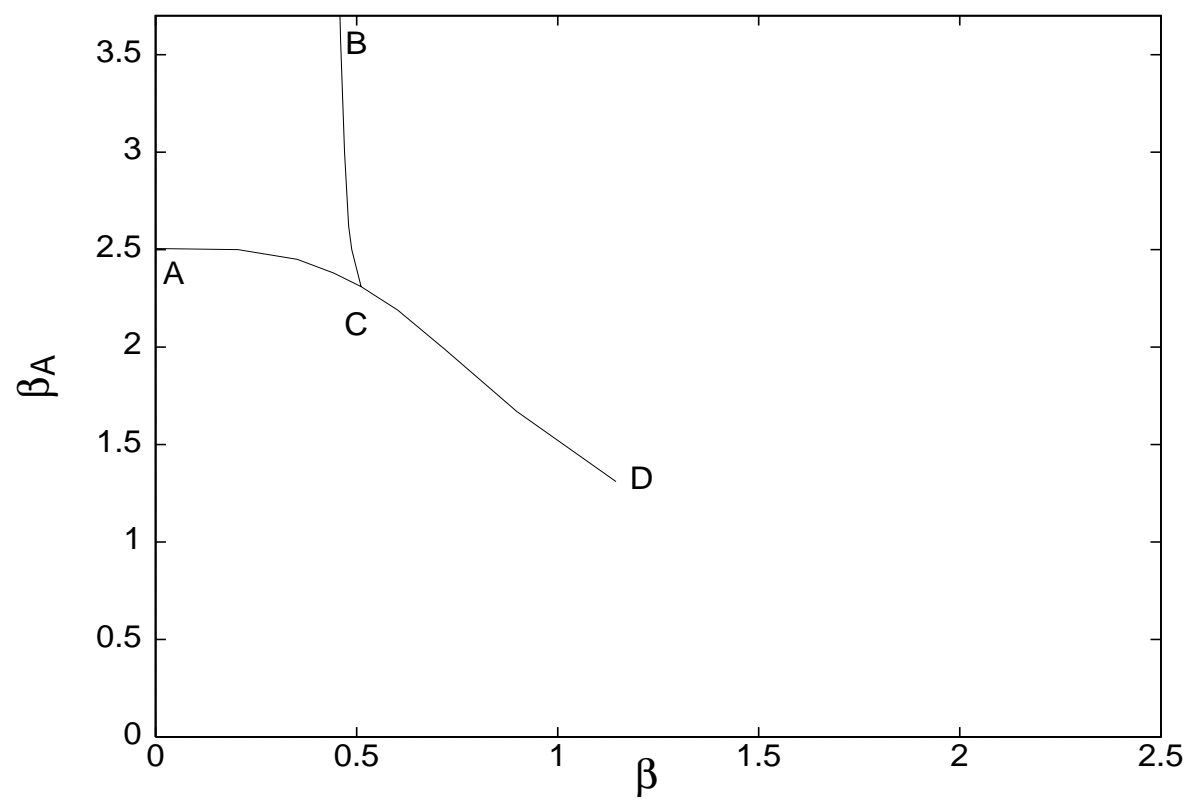

Figure 1: The phase diagram for the action (11), showing the first order bulk phase transition lines. Taken from Ref. [6] but with the endpoint $\mathbf{D}$ as obtained in Ref. [10].

Bhanot and Creutz[6] found that the lattice theory defined by the extended action of eq.(11) has a rich phase structure, shown in Fig. 1. Similar results were obtained for the Villain action (2) by Halliday and Schwimmer [7]. In either case, the $\beta=0$ axis describes an $S O(3)$ model which has a first order phase transition, denoted by point $\mathbf{A}$ in Fig. 1. At $\beta_{A}\left(\right.$ or $\left.\beta_{V}\right)=\infty$, the theory reduces to a $Z_{2}$ lattice gauge theory with again a first order phase transition at $\beta^{\text {crit }}=\frac{1}{2} \ln (1+\sqrt{2}) \approx 0.44$ [13]. For both the mixed actions, these first order transitions extend into the coupling plane, as shown in Fig. 
1 by continuous lines. These lines meet at a triple point $\mathbf{C}$ and continue as a single line of first order transitions which ends at an apparently critical point D. The proximity of $\mathbf{D}$ to the $\beta_{A}=0$ line, which defines the Wilson action, has commonly been held responsible for the abrupt change from the strong coupling region to the scaling region for the Wilson action. It has also been attributed as a possible reason for the dip in the non-perturbative $\beta$-function obtained by Monte Carlo Renormalization Group methods. Indeed, its relative closeness to the $\beta_{A}=0$ line for the $S U(2)$ theory compared to the $S U(3)$ theory has been thought 14 of as a possible reason for the shallower dip in the former case.

The bulk transition in the Villain form of the $\mathrm{SO}(3)$ gauge theory is known [15] to be caused by a condensation of $Z_{2}$ monopoles in the strong coupling phase. Defining $\sigma_{c}=\prod_{p \in \partial c} \sigma_{P}$ for an elementary cube $c$, one finds that $\sigma_{c}=-1$ characterizes a monopole located in $c$. These monopoles are absent in the weak coupling region, and can be suppressed at stronger couplings by adding a term, $\lambda_{M} \sum_{c}\left(1-\sigma_{c}\right)$ to the action (2) and setting $\lambda_{M}$ large. Using this extra term, Ref. [12] demonstrated a clear merging of the second order deconfinement line with a first order bulk transition line for $\lambda_{M}=1$ for the Villain action (2). Moreover, two separate transitions were shown to exist on the same lattice near the merging point, thereby shedding some light on the paradoxes a]-c] above. While pointing to the peculiar role the bulk transitions play in affecting the deconfinement transitions, these results also suggested that $Z_{2}$ electric current loops or vortices have to be suppressed in restoring the universality for the mixed action fully. Defining $\sigma_{l}$ for a link $l \equiv(x, \hat{\mu})$ at a point $x$ on the lattice in the $\mu$ th direction as a product of $\sigma_{P}$ of all those plaquettes which share the link $l$, i.e, $\sigma_{l}=\prod_{p \in \hat{\partial} l} \sigma_{P}$, one finds that $\sigma_{l}=-1$ signals the link to be a part of an $Z_{2}$ electric current loop. Adding further a term $\lambda_{E} \sum_{l}\left(1-\sigma_{l}\right)$ to the action (2) in addition to the monopole suppression term above, Refs. [11, 12] showed that universality is restored in the entire coupling plane for large $\lambda_{E}$.

While a similar mechanism is expected to work for the Bhanot-Creutz mixed action (II) as well, it is clear that both the monopole and vortex suppression terms added above do not depend on the gauge variables $U_{\mu}(x)$ directly and have to be generalized suitably for addition to $S_{B C}$. One possible way is to define $\sigma_{c}$ and $\sigma_{l}$ by replacing $\sigma_{P}$ in them by $\operatorname{sign}\left(\operatorname{Tr}_{F} U_{P}\right)$. Thus the mixed action with chemical potentials for these monopoles and vortices in that case is given by 


$$
\begin{aligned}
S_{B C, S}= & \sum_{P}\left(\beta\left(1-\frac{1}{2} \operatorname{Tr}_{F} U_{P}\right)+\beta_{A}\left(1-\frac{1}{3} \operatorname{Tr}_{A} U_{P}\right)\right) \\
& +\lambda_{M} \sum_{c}\left(1-\sigma_{c}\right)+\lambda_{E} \sum_{l}\left(1-\sigma_{l}\right)
\end{aligned}
$$

where $\sigma_{c}=\prod_{p \in \partial c} \operatorname{sign}\left(\operatorname{Tr}_{F} U_{P}\right)$ and $\sigma_{l}=\prod_{p \in \hat{\partial} l} \operatorname{sign}\left(\operatorname{Tr}_{F} U_{P}\right)$. Comparing the naive classical continuum limit of eq. (3) with the standard $S U(2)$ Yang-Mills action, one obtains

$$
\frac{1}{g_{u}^{2}}=\frac{\beta}{4}+\frac{2 \beta_{A}}{3}
$$

Here $g_{u}$ is the bare coupling constant of the continuum theory. Since the asymptotic scaling equation for the above mixed action with additional (irrelevant) couplings $\lambda_{M}$ and $\lambda_{E}$ can be easily written down in terms of $g_{u}$, it is clear that the introduction of a non-zero $\beta_{A}, \lambda_{M}$, or $\lambda_{E}$ does not affect the continuum limit: the theory for each $\beta_{A}$, including the usual Wilson theory for $\beta_{A}=0.0$, flows to the same critical fixed point, $g_{u}^{c}=0$, in the continuum limit for all $\lambda_{M}$ and $\lambda_{E}$ and has the same scaling behavior near the critical point.

One sees that even for $\beta_{A}=0$ eq. (3) corresponds to a modified Wilson action with different densities of the monopoles and vortices depending on the values of $\lambda_{M}$ and $\lambda_{E}$ respectively. By analogy with the works of Refs. [11, 12] for the Villain action, one expects to eliminate all bulk transition lines in Fig. 1 by setting $\lambda_{M}$ and $\lambda_{E}$ large. In particular, the critical point $\mathbf{D}$ is expected to be absent in that case, leading perhaps to a lot smoother transition from the strong coupling region to the scaling region for those values. It has to be stressed though that universality has to be tested afresh for eq.(3) even for $\beta_{A}=0$ to be sure that the above naive argument about the $\lambda_{M}$ and $\lambda_{E}$ terms being irrelevant is correct. This is what we do in the following by determining a critical index of the deconfinement phase transition. We then check whether the passage to scaling is affected by studying the deconfinement transition as a function of the temporal lattice size.

It is interesting to note that no similar change occurs on the fundamental axis $\left(\beta_{V}=0\right)$ in the case of the Villain action since the variables $\sigma_{P}$ are decoupled from the gauge variables in that case and can be integrated out 
exactly for any observable depending solely on $U_{\mu}(x)$. However, even in that case the $\sigma_{c}$ and $\sigma_{l}$ could have been defined in terms of $\operatorname{sign}\left(\operatorname{Tr}_{F} U_{P}\right)$ and similar results as we obtain below would be obtained.

We studied the deconfinement phase transition on $N_{\sigma}^{3} \times N_{\tau}$ lattices by monitoring its order parameter and the corresponding susceptibility for $N_{\tau}=$ 4, 5, 6 and 8 and $N_{\sigma}=8,10,12,14,15$, and 16. We used the simple Metropolis algorithm and tuned it to have an acceptance rate $\sim 40 \%$. The expectation values of the observables were recorded every 20 iterations to reduce the autocorrelations. Errors were determined by correcting for the autocorrelations and also by the jack knife method. The observables monitored were the average plaquette, $\mathrm{P}$, defined as the average of $\operatorname{Tr}_{F} U_{P} / 2$ over all independent plaquettes, and the absolute value of the average of the deconfinement order parameter [16], $L(\vec{n})$, over the three dimensional lattice spanned by $\vec{n}$, where $L$ is defined by

$$
L(\vec{n})=\frac{1}{2} \operatorname{Tr}_{F} \prod_{\tau=1}^{N_{\tau}} U_{0}(\vec{n}, \tau) .
$$

Here $U_{0}(\vec{n}, \tau)$ is the timelike link at the lattice site $(\vec{n}, \tau)$. In order to monitor the nature of deconfinement and bulk phase transitions, we also define the susceptibilities for both $|L|$ and $\mathrm{P}$ :

$$
\begin{aligned}
\chi_{|L|} & =N_{\sigma}^{3}\left(\left\langle L^{2}\right\rangle-\langle|L|\rangle^{2}\right) \\
\chi_{P} & =6 N_{\sigma}^{3} N_{\tau}\left(\left\langle P^{2}\right\rangle-\langle P\rangle^{2}\right) .
\end{aligned}
$$

According to the finite size scaling theory [17], the peak of the $|L|$ (or plaquette) susceptibility at the location of the deconfinement (or bulk) transition should grow on $N_{\sigma}^{3} \times N_{\tau}$ lattices like

$$
\chi_{|L| \text { or } P}^{\max } \propto N_{\sigma}^{\omega},
$$

for fixed $N_{\tau}$. For a second order transition, $\omega \equiv \gamma / \nu$, where $\gamma$ and $\nu$ characterize the growth of the $|L|$ (plaquette)-susceptibility and the correlation length near the critical temperature (coupling)on an infinite spatial lattice. If the phase transition were to be of first order instead, then one expects [18] the exponent $\omega=3$, corresponding to the dimensionality of the space. In addition, of course, the average $|L|$ or plaquette is expected to exhibit a sharp, or even discontinuous, jump and the corresponding probability distribution should show a double peak structure in case of a first order phase 
transition. Such an analysis of the $|L|$-susceptibility for the Wilson action, where only $\beta$ is nonzero in eq. (3), yielded [8] an exponent $\omega=1.93 \pm 0.03$, in good agreement with the corresponding value $(1.965 \pm 0.005)$ for the three dimensional Ising model, and the universality conjecture 9]. Universality of the continuum limit of lattice gauge theories predicts a similar deconfinement transition belonging to the same universality class as the three dimensional Ising model for all values of $\beta_{A}, \lambda_{M}$ and $\lambda_{E}$.

\section{RESULTS OF THE SIMULATIONS}

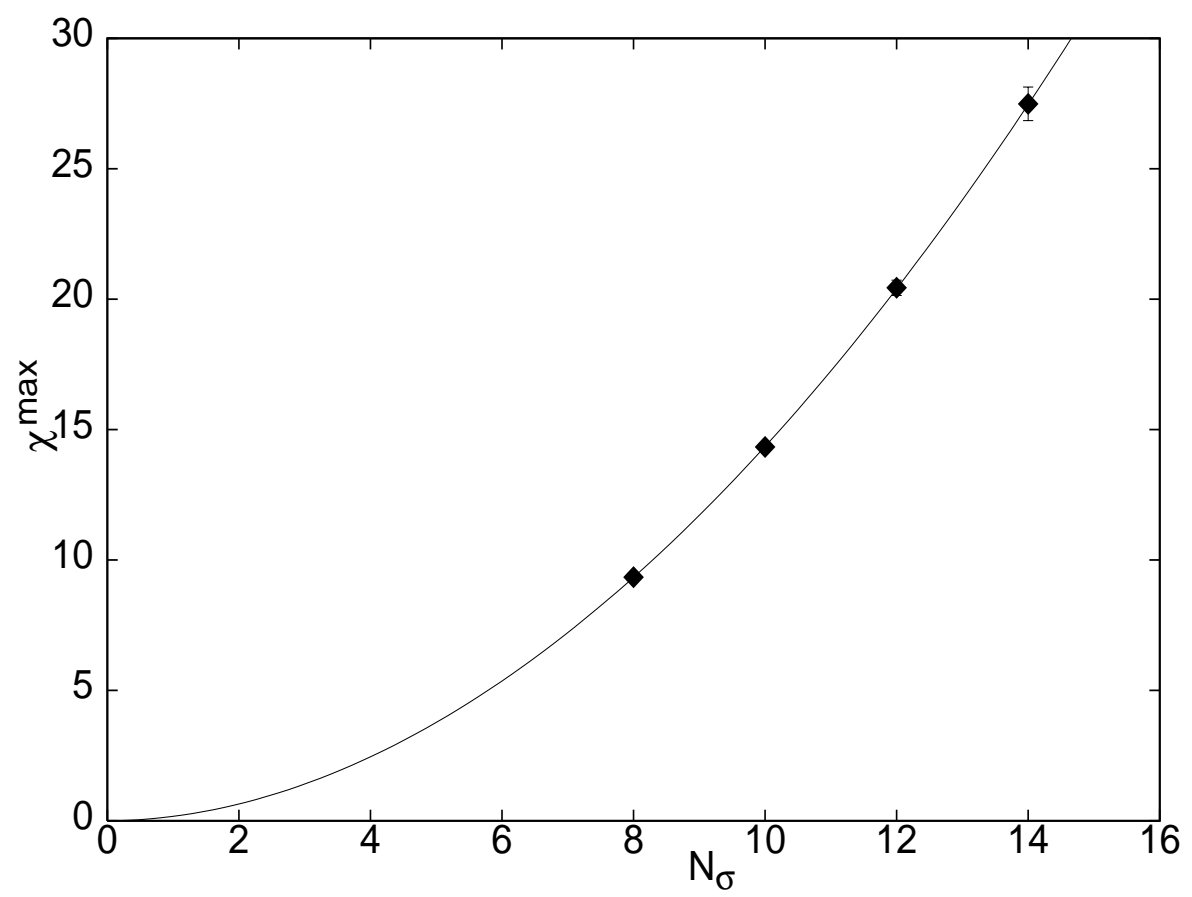

Figure 2: Variation of the peak height of $|L|$-susceptibility with spatial size $N_{\sigma}$ for fixed $N_{\beta}=4$. The curve is a fit to the eq.(8).

In view of the fact that even for $\beta_{A}=0$, the action (3) differs from the Wilson action in a non-trivial manner for nonzero $\lambda_{M}$ and $\lambda_{E}$, we concentrate 
here on $\beta_{A}=0$ and large $\lambda_{M}$ and $\lambda_{E}$. Our aim is to check the impact of the suppression of monopoles and vortices, defined by the $\operatorname{sign}\left(\operatorname{Tr}_{F} U_{P}\right)$ as above, on the critical exponent $\omega$ on lattices with fixed $N_{\tau}$. We then wish to study the scaling behaviour of the deconfinement transition by varying $N_{\tau}$. We chose $\lambda_{M}=1$ and $\lambda_{E}=5$ throughout this work. Variations with respect to these as well as $\beta_{A}$ should in principle be investigated although one would expect the results to display universality for sufficiently large $\lambda_{M}$ and $\lambda_{E}$ if the $\beta_{A}=0$ results do so.

$$
3.1 N_{\tau}=4
$$

The deconfinement phase transition on $N_{\sigma}^{3} \times 4$ lattices for $N_{\sigma}=8,10$, 12 and 14 lattices was studied by first making short hysteresis runs on the smallest lattice to look for abrupt or sharp changes in both the average plaquette $\langle P\rangle$ and the order parameter $\langle|L|\rangle$. In the region of strong variation of $\langle|L|\rangle$, longer runs were made to check whether the $|L|$-susceptibility exhibits a peak. Histogramming technique [19] was used to extrapolate to nearby couplings for doing this. A fresh run was made at the $\chi_{|L|}$ peak position and the process repeated until the input coupling for the run was fairly close to the output peak position of the susceptibility. The same procedure was used for the bigger lattices also but by starting from the $\beta_{c}$ of the smaller lattice. No peak was found in the vanishingly small plaquette susceptibility throughout, suggesting a lack of any nearby bulk transition. This should be contrasted[20] with the results for the $\lambda_{M}=\lambda_{E}=0$, which is known to exhibit a peak. Typically 100-200 thousand measurements (2-4 million Monte Carlo iterations) were used to estimate the magnitude of the peak height and the peak location for each $N_{\sigma}$. Table 1 lists our final results for all the $N_{\sigma}$ used. The errors on $\beta_{c}$ were estimated by varying the bin size while those for $\chi^{\max }$ were taken to be the maximum of the errors for all bin sizes. Fitting the peak heights in Table 1 to eq.(8), we obtained

$$
\omega=1.93 \pm 0.03
$$

Fig. 2 displays the very good quality of the fit. The critical exponent $\omega$ is in excellent agreement with the values for both the standard Wilson action 8] and the 3-dimensional Ising model quoted in previous section. Fitting the peak locations $\beta_{c, N_{\sigma}}$ by the usual finite size scaling expression,

$$
\beta_{c, N_{\sigma}}=\beta_{c, \infty}+B / N_{\sigma}^{1 / \nu},
$$


where $\nu=0.63$ is the correlation length exponent for the 3-dimensional Ising model and $B$ is a constant, we obtained $\beta_{c, \infty}=1.326 \pm 0.006$, which is shifted by about one from the corresponding value for the $\lambda_{M}=\lambda_{E}=0$ case which is $2.2986 \pm 0.0006$ [8]. Inspired by the agreement of $\omega$ above, we assumed universality to be true for $\nu$ as well in eq. (10). However, any reasonable variation of $\nu$ between 0.33 and 1 changes the infinite volume extrapolation for $\beta_{c}$ by a few $(\sim 2-3)$ per cent only. Thus the shift $\delta \beta=\beta_{c}^{\text {Wilson }}-\beta_{c}=$

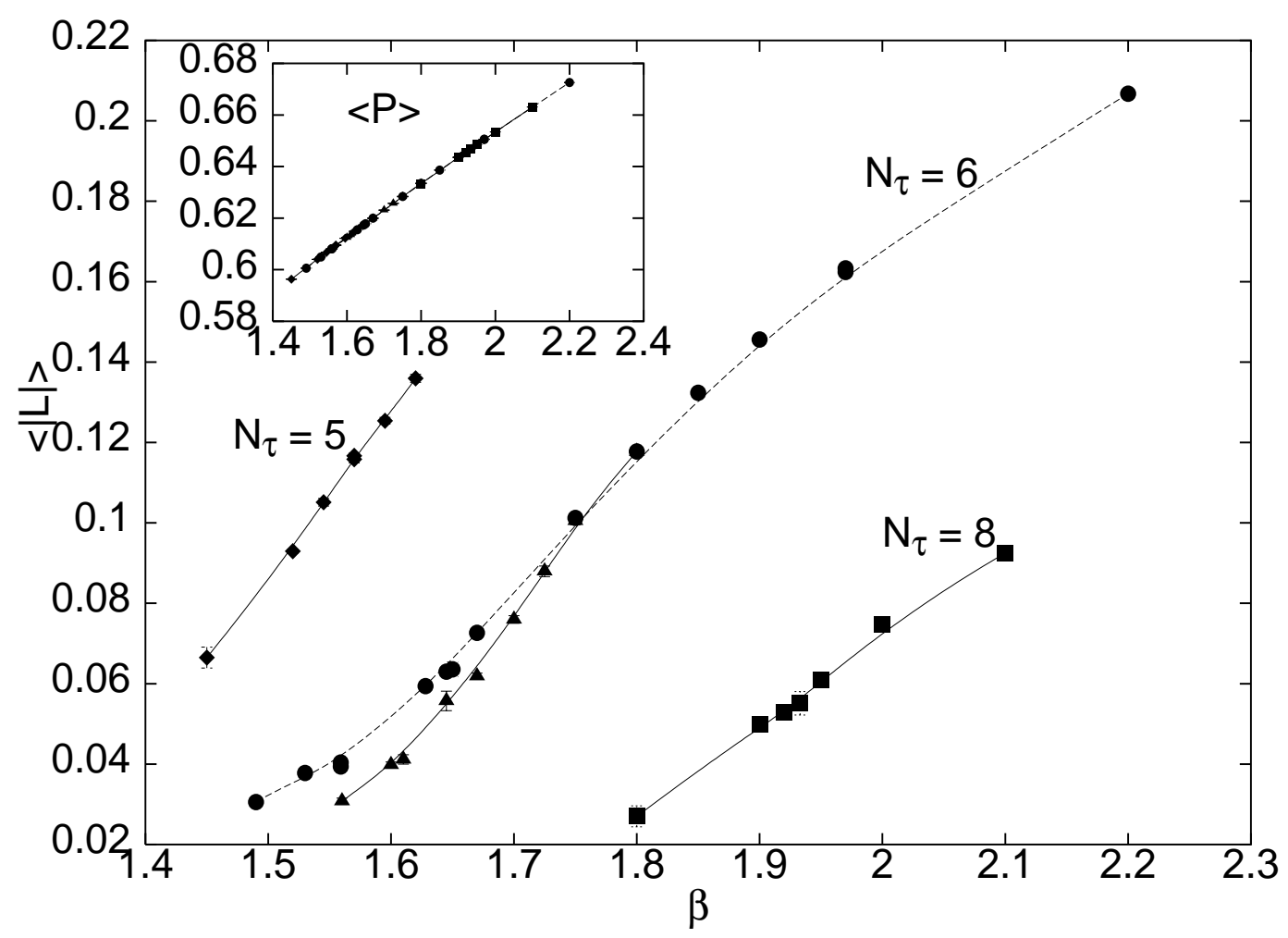

Figure 3: The deconfinement order parameter $\langle|L|\rangle$ as a function of $\beta$ for various temporal lattices $N_{\tau}$. For $N_{\tau}=6$, the circles (triangles) correspond to a $12^{3}\left(15^{3}\right)$ spatial lattice. The inset displays average plaquette $\langle P\rangle$ on all $N_{\tau}$ as a function of $\beta$. The lines are smooth extrapolations of the data to guide the eye. 
0.97 appears to be dominantly due to nonzero $\lambda_{M}$ and $\lambda_{E}$, i.e, suppression of monopoles and vortices.

$$
3.2 N_{\tau}=5,6 \text { and } 8
$$

In order to minimize finite spatial volume effects, we chose to work with $N_{\sigma} \geq 2 N_{\tau}$ always, as seen in sec. 3.1. Consequently, the full 4-volume $N_{\sigma}^{3} N_{\tau}$ increased rapidly as we increased $N_{\tau}$. This resulted in progressive shrinking of the coupling interval in which the histogramming technique was reliable. We therefore used many longer runs in the region of strong variation of $\langle|L|\rangle$ to obtain the susceptibility directly and used the histogramming only for the finer determination of the critical coupling. Fig. 3 exhibits our results for $\langle|L|\rangle$ and $\langle P\rangle$ (shown in the inset) as a function of $\beta$ for $N_{\tau}=5,6$ and 8. A deconfinement phase transition is clearly visible for all of them. The behaviour of the order parameter for two spatial volumes $12^{3}$ and $15^{3}$ for $N_{\tau}$ $=6$ also supports the existence of a transition. This is more clearly seen in the corresponding $\chi_{|L|}$ determinations, shown in Fig. 4 . The plaquette, on the other hand, describes a smooth and unique curve for all $N_{\tau}$ and $N_{\sigma}$ values. As the inset in Fig. 33 shows, plaquette values for all these lattices fall on the same curve, indicating an absence of any bulk transition. Tables 2 and 3 list the estimated maxima of $\chi_{|L|}$ for $N_{\tau}=5$ and 6 for two different spatial volumes along with the corresponding peak locations. As seen in Fig. 4 they are rather close to the input $\beta$ at which the long runs were made. Using our value for $\omega$ from eq.(9), determined for $N_{\tau}=4$, and the peak height for the smaller spatial volume, the $\chi^{\max }$ on the bigger lattice can be predicted. These predictions are listed in the respective tables in the last column and can be seen to be in very good agreement with the direct Monte Carlo determinations. Alternatively, one can fit eq.(8) to the peak heights in Tables 2 and 3 and determine $\omega$ again :

$$
\begin{aligned}
& \omega=1.99 \pm 0.17 \text { for } N_{\tau}=5, \text { and } \\
& \omega=1.80 \pm 0.14 \text { for } N_{\tau}=6 .
\end{aligned}
$$

Both these determinations agree with the canonical values as well as our own value in eq.(9). Not only is the universality of the deconfinement phase transition thus verified on three different temporal lattice sizes, but it also confirms that the same physical phase transition is being simulated on them, 


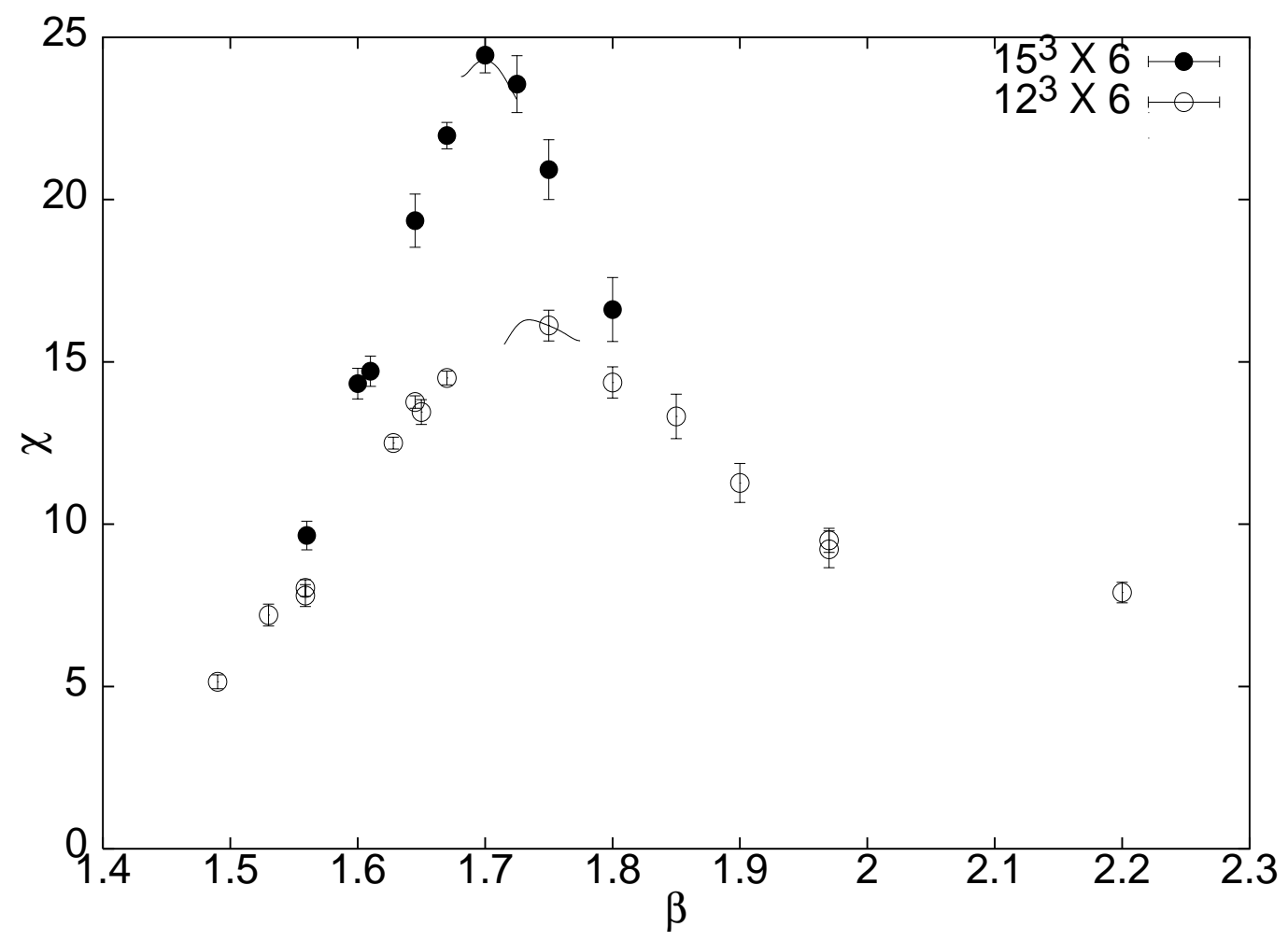

Figure 4: The $|L|$-susceptibility as a function of $\beta$ on lattices with $N_{\tau}=6$. The continuous lines are extrapolations using the histogramming technique. 
thus approaching the continuum limit of $a \rightarrow 0$ in a progressive manner by keeping the transition temperature $T_{c}$ constant in physical units.

\subsection{Scaling of $T_{c}$}

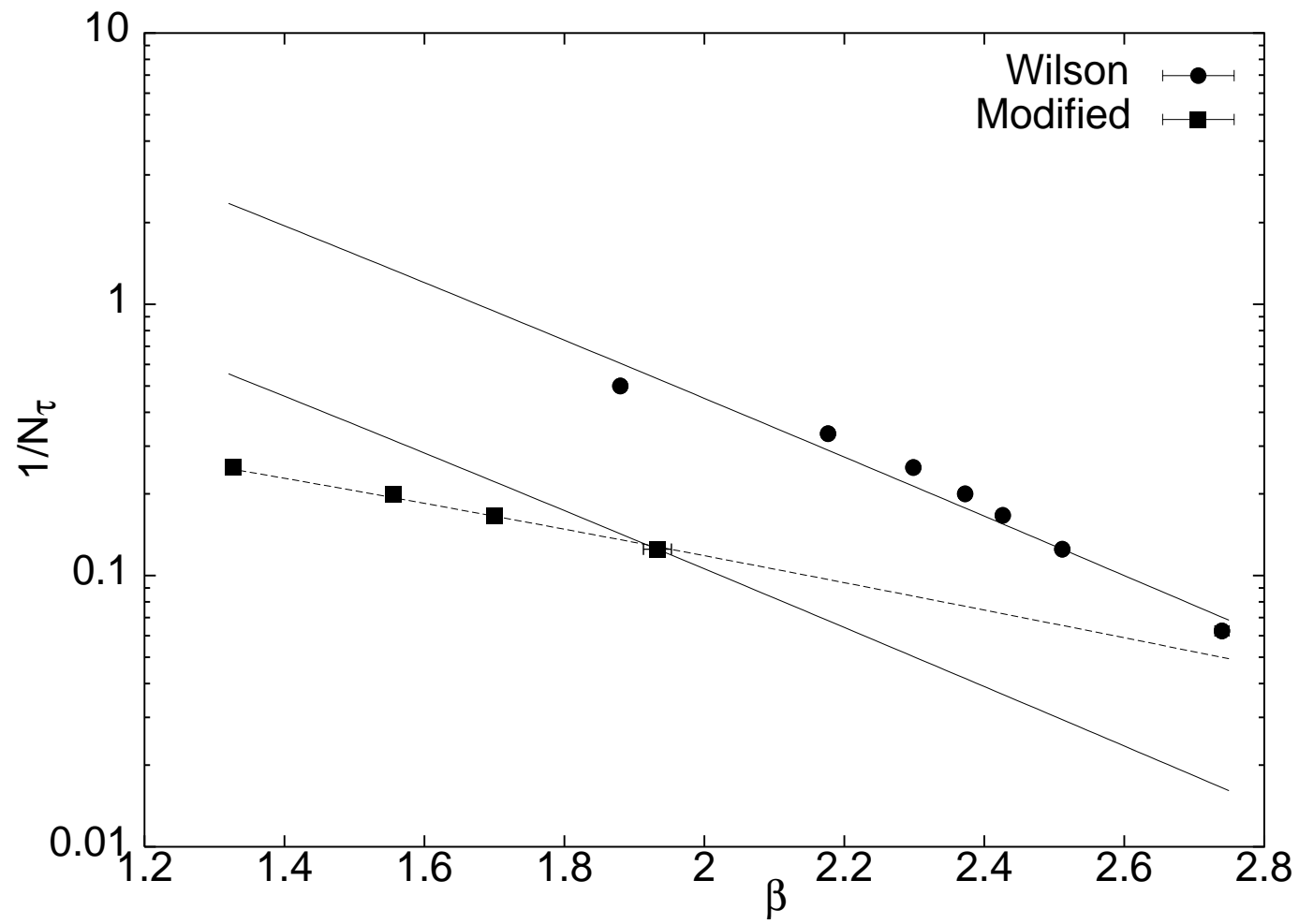

Figure 5: $1 / N_{\tau}$ as a function of $\beta_{c}$. The squares are from this work while circles are from Ref. [21]. The full lines depict the 2-loop asymptotic scaling relation of eq.(12), normalized at $N_{\tau}=8$ in both cases. The dashed line denotes eq.(14), normalized the same way.

Table 4 lists the $\beta_{c}$ (estimated by extrapolating to infinite volume whenever possible) for all the $N_{\tau}$ values we used. The corresponding values for the usual Wilson action, i.e., $\lambda_{M}=0$ and $\lambda_{E}=0$ case, are also given in Table 
4 along with the shifts caused by switching on these two couplings. The shifts decrease with increasing $N_{\tau}$ but nevertheless remains sizeable even for the largest lattice we used. Their decrease smoothens the approach to the scaling limit, as we shall see below. Fig. 5 shows $a T_{c}=N_{\tau}^{-1}$ as a function of the corresponding critical $\beta$ for both our simulations with suppression of monopoles and vortices and the standard Wilson action (without any such suppression). The latter are taken from the compilation of Ref. 21]. The full lines in the figure show the 2-loop asymptotic scaling relation

$$
a T_{c}=\frac{1}{N_{\tau}} \propto\left(\frac{4 b_{0}}{\beta}\right)^{-b_{1} / b_{0}^{2}} \exp \left(-\frac{\beta}{8 b_{0}}\right)
$$

where

$$
b_{0}=\frac{11}{24 \pi^{2}}, \text { and } \quad b_{1}=\frac{17}{96 \pi^{4}}
$$

are the first two coefficients of the perturbative $\beta$-function for the $S U(2)$ Yang-Mills theory. The curve in each case was normalized to pass through the $N_{\tau}=8$ data point. The dashed line describes a 'phenomenological' scaling equation which is similar to the eq.(12) but with the exponent increased by a factor of two:

$$
a T_{c}=\frac{1}{N_{\tau}} \propto\left(\frac{4 b_{0}}{\beta}\right)^{-b_{1} / b_{0}^{2}} \exp \left(-\frac{\beta}{4 b_{0}}\right) .
$$

One sees deviations from asymptotic scaling for both the Wilson action and our action with suppression of monopoles and vortices. The deviations for the same range of $N_{\tau}$ seem larger for our action but then one is also considerably deeper in the strong coupling region of the Wilson action where one a priori would not have even expected any scaling behaviour. As the agreement of our results with the dashed line of eq.(14) in Fig. 5 shows, scaling may hold in this region of $\beta$ for the suppressed action, since the relation between $a$ and $\beta$ in this region (or $g^{2}$ ) is similar to the asymptotic scaling relation, differing only in the exponent which will cancel in dimensionless ratios of physical quantities. It is clear that as $\beta \rightarrow \infty$, the difference between the two actions must vanish. The shifts in Table 4 do show such a trend although the limiting point is not reached by $N_{\tau}=8$ definitely. It seems likely though that the trend of evenly spaced transition points for our action will continue and the dashed line traced by its transition points will merge with the Wilson 
action by $N_{\tau} \sim 25$ or so, as suggested by its approach to the data for the Wilson action. If this were to be so, a much smoother approach to continuum

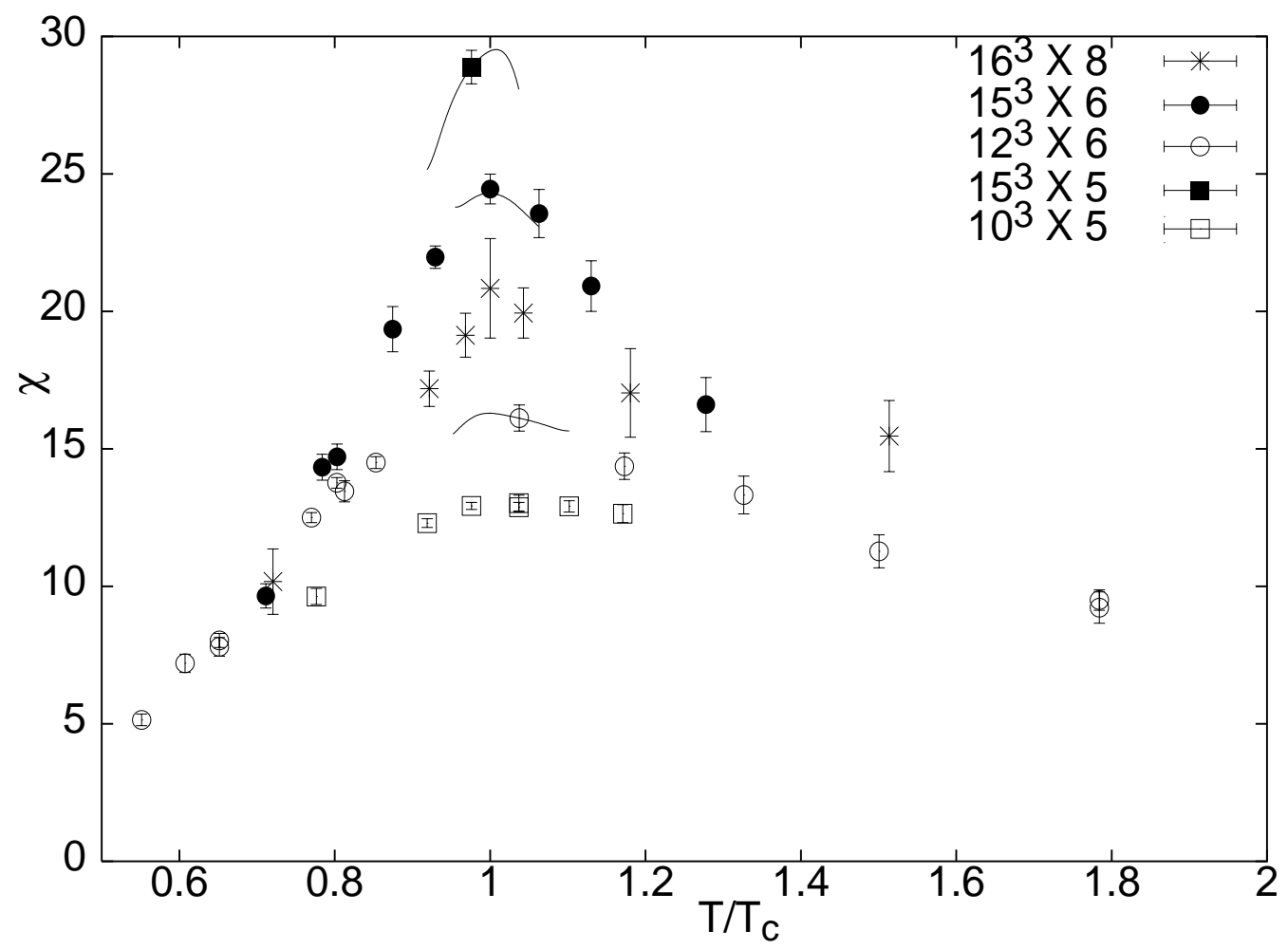

Figure 6: Same as Fig. 1 but as a function of $T / T_{c}$ and on additional lattices, as indicated.

limit is to be expected after the suppression of monopoles and vortices. In particular, one expects that dimensionless ratios of physical quantities at the deconfinement phase transition couplings should be constant, already from $\beta \sim 1.33$, which is the transition point for the $N_{\tau}=4$.

One possible interpretation of the results in Fig. 5 is that the proximity of the point $\mathbf{D}$ in Fig. [ 1 for the usual Wilson action causes the nontrivial curvature visible in the data for the Wilson action, and consequently its approach to the continuum limit is not so smooth. A strong suppression 
of monopoles and vortices, as performed here, eliminates $\mathbf{D}$, resulting in a smoother approach to scaling. Of course, for very small lattice spacings ( or large $\beta$ ), no significant difference between the two will be seen but for sizeable values of the cut-off one may expect the action with suppression to exhibit a better and smoother approach to the continuum limit. We intend to check this by measuring the glueball spectrum at the critical couplings for $N_{\tau}=$ 4-8. In the meantime, one can try to check this hypothesis by using eq. (14) to convert our $\chi(\beta)$ results to $\chi\left(T / T_{c}\right)$, i.e, as a function of a dimensionless ratio for various $N_{\tau}$. Fig. 6 depicts the susceptibility $\chi$ as a function of $T / T_{c}$ on lattices with $N_{\tau}=5,6$ and 8. Ideally one would have expected all susceptibility data for the same physical volume to fall on the same curve for different $N_{\tau}$. These are the three lowest curves with physical volume $8 T^{-3}$ but with $N_{\tau}=5,6$ and 8 . Unfortunately, the order parameter $\langle|L|\rangle$ is not ultra-violet safe; it contains divergent contributions in the continuum, making it $N_{\tau}$ (or $a$ )-dependent even as a function of $T / T_{c}$. Consequently, the corresponding $\chi^{\prime}$ 's are close but not on any universal curve. On the other hand, an increase in physical volume to $15.6 T^{-3}$ (the $15^{3} \times 6$ data) and $27 T^{-3}$ (the $15^{3} \times 5$ data) does seem to sharpen the susceptibility peak progressively, as expected. Although no quantitative analysis can be done meaningfully due to the cut-off dependence of the order parameter itself, the results do show the right trend and thus support a possible scaling in the coupling region of these data points.

\section{SUMMARY AND DISCUSSION}

The phase diagram of the mixed action of eq. (四) in the fundamental and adjoint couplings, $\beta$ and $\beta_{A}$, has been a crucial input in understanding many properties of the $S U(2)$ and $S U(3)$ lattice theories and their continuum limits. The cross-over to the scaling region from the strong coupling region, as well as the dip in the non-perturbative $\beta$-function have been attributed to the location of the end point $\mathbf{D}$ of the line of bulk first order phase transition. In fact, even the relative shallowness of the dip for the $S U(2)$ case compared to the $S U(3)$ case is thought to be due to the closeness of the corresponding end point to the $\beta_{A}=0$ Wilson axis. Adding extra irrelevant terms to the action one obtains the modified action of eq.(3) in which monopoles and vortices can be suppressed by setting the additional couplings to large values. 
Based on the works 11, 12 for Villain action, one expects the phase diagram to change completely in that case. In particular, no phase transition lines or their critical end point $\mathbf{D}$ will be there, causing a smoother transition from the strong coupling region to the scaling region.

In this paper we studied the deconfinement phase transition on the fundamental axis in the $\left(\beta, \beta_{A}\right)$ coupling plane but with $\lambda_{M}=1$ and $\lambda_{E}=5$, i.e., with strong suppression of monopoles and vortices. Our finite size scaling analysis yielded $1.93 \pm 0.03$ for the critical exponent $\omega \equiv \gamma / \nu$ for lattices with $N_{\tau}=4$. This value is in excellent agreement with that 8 for the Wilson action and the three dimensional Ising model, thus verifying the naive universality of the modified action. However, as a result of the suppression, the critical coupling is shifted by about unity compared to the Wilson case. Our results on $N_{\tau}=5$ and 6 also yielded similar values for $\omega$ albeit with larger errors, confirming that the same physical transition was being studied this way as a function of the lattice cut-off, $a$. While the $a T_{c}=N_{\tau}^{-1}$ was found to vary slower than expected from the asymptotic scaling relation (12) for $N_{\tau}$ $=4-8$, the data did obey a similar relation with a factor of two larger exponent. A straightforward extrapolation suggests the results from the modified action will merge with those of Wilson action for large $N_{\tau}$ ( of about $\sim 25$ ), as expected in the limit of vanishing lattice spacing $a$. This suggests that the suppression makes the approach from the strong coupling side to the scaling side much smoother than that for the unsuppressed Wilson action, allowing us to simulate the theory at smaller $\beta$. It will be interesting to see if dimensionless ratios of physical quantities such as glueball masses or string tension with $T_{c}$ are constant in the range of critical couplings explored here. Since the phase diagram for $S U(3)$, and indeed for $S U(N)$ lattice gauge theories, is similar and the same mechanism is expected to work for them, it will also be interesting to study such suppression in those theories as well. However, additional possibilities for topological objects may add further complications and may make it necessary to suppress them as well.

\section{ACKNOWLEDGMENTS}

It is a pleasure to acknowledge interesting discussions with Sourendu Gupta. 


\section{References}

[1] K. Wilson, Phys. Rev. D10, 2445 (1974).

[2] R. V. Gavai, M. Grady and M. Mathur Nucl. Phys. B423, 123 (1994).

[3] M. Mathur, R. V. Gavai, Nucl. Phys. B448, 399 (1995); Nucl. Phys. B (PS) 42, 490 (1995).

[4] R. V. Gavai and M. Mathur, Phys. Rev. D 56, 32 (1997).

[5] P. W. Stephenson, hep-lat/9604008.

[6] G. Bhanot and M. Creutz, Phys. Rev. D24, 3212 (1981).

[7] L. Caneschi, I. G. Halliday and A. Schwimmer, Nucl. Phys. B200, 409 (1982).

[8] J. Engels, J. Fingberg and M. Weber, Nucl. Phys. B332, 737 (1990); J. Engels, J. Fingberg and D. E. Miller, Nucl. Phys. B387, 501 (1992).

[9] B. Svetitsky and L. G. Yaffe, Nucl. Phys. B210[FS6], 423 (1982).

[10] R. V. Gavai, Nucl. Phys. B474, 446 (1996).

[11] R. V. Gavai and M. Mathur, Phys. Lett. B458, 331 (1999).

[12] Saumen Datta and R. V. Gavai, Nucl. Phys. B (PS) 83-84, 366 (2000); Phys. Rev. D62, 054512 (2000).

[13] F. J. Wegner, J. Math. Phys. 12, 2259 (1971).

[14] A. Patel, S. Otto and R. Gupta, Phys. Lett. 159B, 143 (1985);

U. Heller and F. Karsch, Phys. Rev. Lett. 54, 1765 (1985).

[15] I. G. Halliday and A. Schwimmer, Phys. Lett. 102B, 337 (1981).

[16] L. McLerran and B. Svetitsky, Phys. Rev. D24, 450 (1981). 
[17] M. N. Barber, in Phase Transitions and Critical Phenomena, vol. 8, Ed. C. Domb and J. L. Lebowitz (Academic Press, New York, 1983) p. 146.

[18] M. S. Challa, D. P. Landau and K. Binder, Phys. Rev. B34, 1841 (1986).

[19] A. M. Ferrenberg and R. H. Swendsen, Phys. Rev. Lett. 61, 2635 (1988).

[20] J. Engels and T. Scheideler, Phys. Lett. B394, 147 (1999); M. Nauenberg, T. Schalk and R. Brower, Phys. Rev. D24, 548 (1981).

[21] J. Fingberg, U. Heller and F. Karsch, Nucl. Phys. B392, 493 (1993). 
Table 1

The values of $\beta$ at which long simulations were performed on $N_{\sigma}^{3} \times 4$ lattices, $\beta_{c}$ and the height of the $|L|$-susceptibility peak, $\chi_{|L|}^{\max }$.

\begin{tabular}{|c|c|c|c|}
\hline$N_{\sigma}$ & $\beta$ & $\beta_{c, N_{\sigma}}$ & $\chi_{|L|}^{\max }$ \\
\hline \hline 8 & 1.37 & $1.366(7)$ & $9.34 \pm 0.07$ \\
\hline 10 & 1.344 & $1.360(5)$ & $14.34 \pm 0.11$ \\
\hline 12 & 1.331 & $1.345(2)$ & $20.44 \pm 0.29$ \\
\hline 14 & 1.34 & $1.343(2)$ & $27.48 \pm 0.64$ \\
\hline \hline
\end{tabular}


Table 2

Same as Table 1 but for on $N_{\sigma}^{3} \times 5$ lattices.

\begin{tabular}{|c|c|c|c|c|}
\hline$N_{\sigma}$ & $\beta$ & $\beta_{c, N_{\sigma}}$ & $\chi_{|L|}^{\max }$ & $\chi_{\text {predicted }}^{\max }$ \\
\hline \hline 10 & 1.545 & $1.570(5)$ & $13.17 \pm 0.17$ & - \\
\hline 15 & 1.545 & $1.558(2)$ & $29.57 \pm 0.77$ & $28.78 \pm 0.41$ \\
\hline \hline
\end{tabular}

Table 3

Same as Table 1 but for on $N_{\sigma}^{3} \times 6$ lattices.

\begin{tabular}{|c|c|c|c|c|}
\hline$N_{\sigma}$ & $\beta$ & $\beta_{c, N_{\sigma}}$ & $\chi_{|L|}^{\max }$ & $\chi_{\text {predicted }}^{\max }$ \\
\hline \hline 12 & 1.75 & $1.735(5)$ & $16.34 \pm 0.45$ & - \\
\hline 15 & 1.70 & $1.702(2)$ & $24.39 \pm 0.41$ & $25.13 \pm 0.70$ \\
\hline \hline
\end{tabular}


Table 4

The values of $\beta_{c}$ at which the deconfinement phase transition takes place on a lattice with temporal extension $N_{\tau}$ for eq. (3) for $\lambda_{M}=1$ and $\lambda_{E}=5$ (column 2) and the usual Wilson action (column 3), taken from Ref. 21. The last column lists the shift $\delta \beta=\beta_{c}^{\text {Wilson }}-\beta_{c}$.

\begin{tabular}{|c|c|c|c|}
\hline$N_{\tau}$ & $\beta_{c}$ & $\beta_{c}^{\text {Wilson }}$ & $\delta \beta$ \\
\hline \hline 4 & $1.327 \pm 0.007$ & $2.2986 \pm 0.0006$ & $0.972 \pm 0.007$ \\
\hline 5 & $1.56 \pm 0.01$ & $2.3726 \pm 0.0045$ & $0.813 \pm 0.011$ \\
\hline 6 & $1.70 \pm 0.01$ & $2.4265 \pm 0.0030$ & $0.727 \pm 0.010$ \\
\hline 8 & $1.933 \pm 0.02$ & $2.5115 \pm 0.0040$ & $0.579 \pm 0.020$ \\
\hline \hline
\end{tabular}

